\title{
The times
}

\section{Mental illness and motor insurance}

\author{
Phillip Brown, Research Fellow, United Medical and Dental School (Guy's Campus), \\ St Thomas' Street, London SE1 9RT
}

Much has been written about mental illness and driving, particularly the possibly increased risk of accidents. There is, however, little awareness of how this affects insurance premiums. It is well known that as a result of recent losses within the business, premiums were increased by $30 \%$ last year, and may be set to rise higher. This has made insuring a car a major part of the cost of running one. It appears that people with mental illnesses are being unfairly treated by motor insurers and therefore financially disadvantaged. I was first made aware of this problem by my involvement in a case.

A woman in her 20s suffered a post-natal depression following the birth of her first child. She was an in-patient on a mother and baby unit for three months and was discharged to her home, taking an anti-depressant. At around this time, she and her husband applied for motor insurance through an insurance broker. The application form included a question about past or present physical or mental illness. This was completed honestly and the recent illness was declared. The company asked for reports from the psychiatrists involved. These reports were very positive, and even went so far as to say that the patient was, in the doctors' opinion, fit to drive. Soon afterwards the insurance company decided to terminate the policy. No explanation was given to the prospective policy holder, but the broker made some informal enquiries of the company, who gave two reasons.

(a) As the proposer had been admitted to hospital she had obviously been seriously depressed.

(b) She might feel suicidal and so drive in an intentionally reckless way.

Both these points seemed disputable. Admission to hospital does not necessarily imply a particular severity of illness, as many other factors must be considered. Also there is evidence that suicide rates are low in the first year after childbirth, despite the high rate of psychiatric morbidity (Appleby, 1991). I wondered if thinking of this kind was typical of motor insurers.

To investigate this I wrote to several large insurance companies asking about their attitudes to mental illness, and their policies in this area. Five companies replied. Three said that they would insure anyone to whom the Driver Vehicle Licensing Authority had issued a licence. They would seek information from various sources (e.g. proposer's doctor, company medical referee, or independent doctor) and might impose restrictions or additional terms. Two companies differed significantly from the rest. Company A stated in a letter:

"Whilst we do not profess to be experts in the field of mental illness, we would normally decline any new proposal from a person currently suffering from a mental illness, and leave these risks to Companies more experienced in this area of the market."

For existing customers who develop mental illness they "try to be more accommodating". They refer to obtaining details and then assessing the situation by "cross referencing with statistics produced by various governing bodies and literature contained in well established medical journals". I wrote further letters attempting to clarify some of these points, but received no reply. Company B wrote:

"As a general rule however we, as with most other insurers, specifically exclude new proposers suffering from any physical or mental disability."

They too are more tolerant of existing policy holders and try to "look at each case on its own merits". In response to another letter they defined "mental disability" as "where a patient is currently receiving medication, or is generally receiving medical supervision, or is currently suffering from a known illness".

Research in this area covers two main topics: the direct effects of mental illnesses, and the effects of prescribed drugs. The literature on medication is relatively large. There is much discussion about how best to test the effects of drugs. Should experiments use healthy volunteers or real patients? Are laboratory tests valid or are low-speed vehicle-handling tests essential? There is good evidence that many drugs prescribed by psychiatrists impair driving but no indication of how this compares to the impairment caused by a legal 79 milligrams percent of alcohol. 
Silverstone (1988) has reviewed the influence of mental illness on driving performance. He concludes:

"It does appear that psychiatric patients, taken overall, have a higher then expected rate of involvement in RTAs (road traffic accidents). But certain conditions are disproportionately represented and by no means all types of psychiatric patient are potentially hazardous behind the wheel."

He finds evidence for increased accident rates in dementia, anxiety states, personality disorders, and alcoholism. There seems to be no evidence of increased risk in schizophrenia, mania, or illicit drug abuse. In the case of depression things are much less clear. As Silverstone states, it is easy to see how those suffering from "severe depression" might be at risk in two ways:

"their slowed responsivity and poor concentration put them at risk from a car handling point of view, while their suicidal ideation may cause them to crash their car in an attempt to end their lives".

His conclusion from the literature is that there may be an association between depression and road traffic accidents. In reviewing largely the same literature Tsuang et al (1985) failed to find evidence for a significant role of suicidal motivation in traffic accidents, but did not address the issue of depression in the absence of suicidal intent. Armstrong \& Whitlock (1980) found that vehicular suicide is more often caused by alcohol and drugs than by severe depression. There is a broad consensus in the literature that alcohol use is of far more importance than any other form of psychiatric illness. It is unclear whether these already tentative findings apply to the whole spectrum of depressive illnesses, or should be restricted to "severe depression".

There are some comparisons to be drawn with physical illnesses. The extensive literature on driving and physical illness focuses on epilepsy and diabetes mellitus. People with epilepsy probably have more traffic accidents, although this is not proven. American authors (Krumholz et al, 1991) wrote:

"Although insurance is generally available to persons with epilepsy, the cost may high and coverage limited."

A British study asked patients with diabetes about their experience of insurance (Frier et al, 1984). Of 147 drivers, 96 had informed their insurer of their diabetes. Of these 14 had been refused motor insurance because of diabetes, and 36 were aware of an increase in premiums. Problems of this kind are clearly not restricted to mental illness.
What are we to think of insurance companies who reject all proposers with any kind of mental illness? To define mental illness so broadly, and to regard it as a uniform source of risk, is clearly not justified by the research evidence. Similar attitudes exist toward some physical illnesses, but at least these conditions are more specifically defined and so likely to affect fewer people. More research could alter attitudes in this area, but like previous work it might be ignored or selectively interpreted. The Association of British Insurers regard the operation of "a very selective underwriting policy" as "commercial decisions for each company to make" (M. J. Tarling, personal communication).

It is the responsibility of the individual driver to inform the Driver and Vehicle Licensing Agency of any "relevant disability". Doctors are not obliged to inform the authorities of a patient's medical condition, but may do so if the individual is likely to be a source of danger to the public. Most motor insurance proposal forms ask about the presence of "mental defect or infirmity". Can we advise our patients to declare illnesses that we would regard as irrelevant, but that we know will jeopardise their application? Dishonesty has its drawbacks, and failure to declare relevant information could lead to insurance policies being invalid, and both civil and criminal law suits. Perhaps, despite these penalties, we should not be surprised that one company reported that "disclosure of a mental health problem tends to be a rarity".

\section{References}

APPLEBY, L. (1991) Suicide during pregnancy and in the first postnatal year. British Medical Journal, 302, $137-140$.

Armstrong, J. L. \& Whitlock, F. A. (1980) Psychiatric illness and motor accidents. Australia and New Zealand Journal of Psychiatry, 14, 53-60.

Frier, B. M., Sullivan, F. M. \& Stewart, E. J. C. (1984) Diabetes and insurance: a survey of patient experience. Diabetic Medicine, 1, 127-130.

KRUMholz, A., Fisher, R. S., Lesser, R. P. \& HaUser, W. A. (1991) Driving and epilepsy. A review and appraisal. Journal of the American Medical Association, 265, 622-626.

SILVERSTONE, T. (1988) The influence of psychiatric disease and its treatment on driving performance. International Clinical Psychopharmacology, 3, (suppl 1) 59-66.

Tsuang, M. T., BoOR, M., Fleming, J.A. (1985) Psychiatric aspects of traffic accidents. American Journal of Psychiatry, 142, 538-546. 